\title{
DA 5505: A Novel Topical Formulation of Terbinafine That Enhances Skin Penetration and Retention
}

\author{
Raj Kumar Thapa, ${ }^{a, \#}$ Sang-Duk Han, ${ }^{b, \#}$ Hyoung Geun Park, ${ }^{b}$ Miwon Son, ${ }^{b}$ Joon Ho Jun, ${ }^{b}$ and \\ Jong Oh Kim*,a \\ ${ }^{a}$ College of Pharmacy, Yeungnam University; 214-1 Daedong, Gyeongsan-si, Gyeongsanbuk-do 712-749, South \\ Korea: and ${ }^{b}$ Dong-A Pharmaceutical Co., Ltd.; Yongin-si, Kyunggi-do 449-905, South Korea. \\ Received February 1, 2015; accepted April 2, 2015; advance publication released online May 9, 2015
}

\begin{abstract}
Topical fungal infections can become severe if left untreated. Efficient treatment modalities for topical fungal infections aid the penetration of antifungal agents deep into viable skin layers. Terbinafine is a fungicidal agent that inhibits ergosterol, an essential fungal component. The main objective of this study was to evaluate skin permeation and retention of a terbinafine-loaded solution containing chitosan as a film former. Comparative assessment of skin permeation and retention was performed using a prepared formulation (DA 5505) and marketed formulations of terbinafine in murine and porcine skin. To mimic fungal infection of skin, keratinized skin was induced in NC/Nga mice. In comparison with the marketed formulations, DA 5505 exhibited significantly better skin permeation. The flux, permeation coefficient, and enhancement ratio of terbinafine were remarkably increased by DA 5505 in comparison with the marketed formulations, and lag time was dramatically reduced. DA 5505 significantly increased cumulative terbinafine retention in viable skin layers in comparison with the marketed solution, suggesting enhanced efficacy. Furthermore, DA 5505 exhibited superior skin permeation in normal skin and keratinized skin. Thus, the DA 5505 formulation has the potential to effectively deliver terbinafine to superficial and deep cutaneous fungal infections.
\end{abstract}

Key words terbinafine; chitosan; topical solution; skin permeation; retention

Superficial cutaneous fungal infections such as candidiasis, pityriasis versicolor, tinea cruris, and dermatophyte infections are very common in developed and developing countries. If left untreated, topical infections can reach the circulation, causing systemic fungal infections that can be fatal. ${ }^{1)}$ Therefore, timely and efficient treatment of superficial cutaneous fungal infections is an important clinical consideration, and topical treatment plays an important role in such treatment.

Topical treatment modalities have several advantages over other delivery methods, including noninvasiveness, ease of use, ability to target infection sites, minimal adverse effects/ drug interactions, improved patient compliance, and reduced treatment costs. ${ }^{2)}$ These characteristics make topical therapy an attractive treatment option for superficial cutaneous fungal infections in comparison with oral drug treatment. Adequate concentrations of antifungal agents at target skin sites can be easily achieved by topical treatment if proper control of drug release and penetration can be achieved. ${ }^{3)}$ In addition, topical antifungal application minimizes adverse effects associated with drug treatment, because much lower blood levels of drugs are produced. ${ }^{1)}$ However, in comparison with other treatment methods, higher oral doses are required to achieve similar local drug concentrations, which can lead to adverse effects. Furthermore, oral administration is associated with severe adverse events, including severe hepatic toxicity, as well as drug-drug interactions. ${ }^{4}$

Terbinafine is a fungicidal agent belonging to the class allylamine with an excellent safety profile that acts by inhibiting ergosterol, an important fungal cell membrane and cell wall component. ${ }^{5,6)}$ It exhibits broad-spectrum activity against fungi, yeast, dermatophytes, and molds. The marketed for- mulation of terbinafine hydrochloride $\left(\mathrm{Lamisil}^{\mathbb{R}}\right)$ is prepared for either oral administration (250 or $500 \mathrm{mg}$ per day) or for topical administration ( $1 \%$ cream, $1 \%$ solution; applied twice daily). ${ }^{7}$ The high efficacy and potency of terbinafine as a treatment for fungal and yeast infections make it a suitable candidate for topical delivery. ${ }^{8}$

Chitosan, a widely used pharmaceutical excipient, is a natural hydrophilic biopolymer that is produced by chitin deacetylation, ${ }^{9)}$ and it possesses several useful characteristics, such as high biocompatibility, nontoxicity, and nonantigenicity. ${ }^{10)}$ The outermost skin layer, known as the stratum corneum, is negatively charged at tight junctions between the cells. Because chitosan is cationic in nature, it can interact with the negatively charged skin, leading to bioadhesiveness and enhancement of percutaneous penetration. ${ }^{11)}$

Antifungal agents used to treat mycotic infections must reach the target site at the desired concentration for effective treatment. Formulations for topical delivery should be able to target the antifungal drug to the viable epidermis and dermis. The rate and extent of drug permeation into the skin and the therapeutic efficacy of a drug following topical administration depend on the nature of the vehicle and the physicochemical properties of the administered drug. ${ }^{72}$ The solution dosage form has been widely used to deliver active pharmaceutical ingredients, because it offers advantages such as ease of application, targeted drug delivery, cost effectiveness, and improved patient compliance. Therefore, in this study, a chitosan-based terbinafine-loaded film forming solution formulation (DA 5505) was developed and its skin permeation and terbinafine retention ability were compared with those of marketed formulations.

\footnotetext{
\# These authors contributed equally to this work.

* To whom correspondence should be addressed. e-mail: jongohkim@yu.ac.kr
} 


\section{Experimental}

Materials Terbinafine was purchased from Dong-Woo Syntech (Eumsung, Korea). Chitosan (Koyo Chemical Co., Sakai-minato, Japan), Eudragit ${ }^{\circledR}$ E PO (Evonik Korea, Seoul, South Korea), polyvinylacetal diethylamino acetate (AEA; Mitsubishi-Kangaku, Nagoya, Japan), octylacrylamide/acrylate copolymer (Demacryl ${ }^{\circledR} 79$ polymer; AkzoNobel, Chicago, IL, U.S.A.), and poly(methylvinylether/maleic acid) (PVM/MA) copolymer (Gantrez ${ }^{\mathrm{TM}}$ polymers; Ashland, Zug, Switzerland) were purchased from commercial sources. DA 5505 was provided by Dong-A Pharmaceutical Co., and Lamisil Once ${ }^{\circledR}$, Lamisil Cream ${ }^{\circledR}$, and Terbinew ${ }^{\circledR}$ gel were purchased from commercial sources. All other chemicals were of analytical grade.

Animals Balb/c nude mice and NC/Nga mice (5-7 weeks old) were purchased from Central Lab Animal Inc. The mice were kept under specific pathogen-free conditions, with food and water provided ad libitum. All animal care and experimental protocols were performed in accordance with the guidelines outlined by the Institutional Animal Ethical Committee of Yeungnam University, South Korea.

HPLC Assay for Terbinafine The quantification of terbinafine was performed with a Shimadzu HPLC system equipped with an SPD-M20A UV/Vis detector, an LC-20AD pump, and a reversed phase $\mathrm{C}_{18}$ column (Capcell pak $\mathrm{C}_{18}$ UG120; $5 \mu \mathrm{m}, 4.6 \mathrm{~mm} \times 150 \mathrm{~mm}$; Shiseido, Japan). LC solution software was used for the HPLC analysis. The mobile phase was a mixture of acetonitrile/tetrahydrofuran $/ 10 \mathrm{~mm}$ phosphate buffer (pH 7.8) (650:150:250), and a flow rate of $2 \mathrm{~mL} / \mathrm{min}$ was used. The analyses were performed at a detection wavelength of $280 \mathrm{~nm}$. Comparison of the HPLC chromatograms of the extracted skin and blank tapes did not reveal any interfering peaks with terbinafine, confirming the excellent selectivity of the method.

Effect of Different Film Formers on Terbinafine Skin Retention The in vitro skin permeation and retention study was conducted using Franz diffusion cells (FCDV15 Labfine; Gyeonggi, South Korea). First, $5 \mathrm{~mL}$ of phosphate buffered saline (PBS) (pH 5.8) was added to the receptor compartment of the Franz diffusion cell. Next, porcine skin was washed with PBS, prehydrated for $30 \mathrm{~min}$, cut into pieces $(3 \mathrm{~cm} \times 3 \mathrm{~cm})$, and attached between the donor and receptor compartments. After the skin was fixed in place, $300 \mathrm{mg}$ equivalents of terbinafineloaded solutions with different film formers were added to the donor compartment, and the drug was allowed to penetrate the skin for $24 \mathrm{~h}$. After $24 \mathrm{~h}$, a $6-\mathrm{mm}$ diameter punch was used to cut the porcine skin into sections, to which $0.5 \mathrm{~mL}$ of $2 \mathrm{~N}$ $\mathrm{NaOH}$ was added. The skin sections were ground for $30 \mathrm{~min}$, after which $1 \mathrm{~mL}$ of ethyl acetate was added and the solution was vortexed for $3 \mathrm{~min}$. The supernatant was collected and $1 \mathrm{~mL}$ of ethyl acetate was added, followed by further centrifugation to obtain the supernatant, which was evaporated. Finally, $1 \mathrm{~mL}$ of the mobile phase was added to the dried supernatant and mixed, and the terbinafine concentration of the resulting solution was analyzed using HPLC.

Determination of Terbinafine Skin Permeation from Different Formulations Skin was obtained from Balb/c nude mice and washed with PBS. The receptor compartment of Franz diffusion cell was filled with $5 \mathrm{~mL}$ of $50 \%$ ethanol. The temperature of the cell was maintained at $37^{\circ} \mathrm{C}$, and the solution was stirred at $300 \mathrm{rpm}$. The mouse skin $(3 \mathrm{~cm} \times 3 \mathrm{~cm})$ was attached between the donor and the receptor compartments. Different marketed formulations or DA 5505 (300 mg terbinafine equivalent) were added to the donor compartment. Aliquots of $200 \mu \mathrm{L}$ were collected from the receptor compartment at predetermined time intervals and replaced with fresh receptor fluid. The samples were centrifuged at $13000 \mathrm{rpm}$ for $10 \mathrm{~min}$, and $100 \mu \mathrm{L}$ of the supernatant was collected and analyzed using HPLC.

The cumulative amount of terbinafine collected in the receptor compartment of the Franz diffusion cell was plotted as a function of time. The flux $\left(J_{\mathrm{ss}}, \mu \mathrm{g} / \mathrm{cm}^{2} \mathrm{~h}\right)$ for each formulation was determined from the slope of the linear portion of the data using regression analysis. The permeation coefficient $\left(K_{\mathrm{p}}\right)$ was determined as the ratio of $J_{\mathrm{ss}}$ to the total donor concentration of the drug. The lag time (LT) was determined from the $x$-intercept of the regression line. The enhancement ratio (ER) for DA 5505 was determined from the ratio of the $K_{\mathrm{p}}$ of DA 5505 to that of the other formulations.

Determination of Terbinafine Skin Retention in Different Skin Layers The receptor compartment of the Franz diffusion cell was filled with $5 \mathrm{~mL}$ of $\mathrm{pH} 5.8$ PBS solution. Porcine skin $(3 \mathrm{~cm} \times 3 \mathrm{~cm})$ was prehydrated for $30 \mathrm{~min}$ and mounted on the diffusion cell, and $300 \mathrm{mg}$ each of Lamisil Once ${ }^{\circledR}$ and DA 5505 were added to the donor compartment and left for $24 \mathrm{~h}$ to allow terbinafine penetration into the skin layers. Upon completion of the experiment, the diffusion cells were dismantled and the receptor phases were removed. The skin surface was washed in running water to discard any residual formulation or drug. The skin surface exposed to the donor compartment was punched out, and the tape stripping method, as reported by Alberti et al. ${ }^{3)}$ and Erdel et al. ${ }^{13)}$ with slight modifications, was used to separate the stratum corneum layers. Briefly, adhesive tape was pressed onto the skin using a roller with constant pressure (rolled back and forth 5 times), and the tape was removed from the skin with a single rapid movement. The non-treated skin surrounding the application site was covered with adhesive tape to ensure that the stripped area consisted of the treated skin. The first piece of tape was discarded to avoid overestimation of the amount of terbinafine in the stratum corneum (SC) and the remaining fragments were merged into 4 fractions: upper SC, lower $\mathrm{SC} 1$, lower SC2, and lower SC3. The stripped samples were then milled and extracted at ambient temperature for $12 \mathrm{~h}$ with continuous shaking. Each sample was filtered through a 0.45 $\mu \mathrm{m}$ membrane filter, and the terbinafine concentration was measured using HPLC as described previously.

Determination of Terbinafine Skin Retention with Different Loading Doses The Lamisil Once ${ }^{\circledR}$ and DA 5505 formulations with different amounts of drug $(30,50,150$, or $300 \mathrm{mg}$ ) were loaded into the donor compartments of the diffusion cells. All other procedures were similar to those used in the study of the effect of different film formers on terbinafine skin retention.

Determination of the Effects of the Formulation on Terbinafine Skin Permeation The effects of the Lamisil Once ${ }^{\circledR}$ and DA 5505 formulations on terbinafine skin penetration were determined. In order to compare the effects of the formulations on drug skin penetration into different skin types, normal and keratinized skin samples were studied. Normal, healthy skin was obtained from Balb/c nude mice, whereas keratinized skin was obtained from $\mathrm{NC} / \mathrm{Nga}$ mice. A protocol 
Table 1. Various Film-Forming Solutions

\begin{tabular}{llcc}
\hline \hline Film-former & \multicolumn{1}{c}{ Composition } & Concentration $(\% \mathrm{w} / \mathrm{v})$ & $\mathrm{pH}$ \\
\hline Lamisil Once ${ }^{\circledR}$ & Acrylate/octylacrylamide copolymer, hydroxypropyl cellulose & N/A & 3.1 \\
Chitosan & Chitosan & 1 & 4.1 \\
Eudragit E & Dimethylaminoethyl methacrylate & 1 & 5.6 \\
AEA & Polyvinylacetal diethylamino acetate & 1 & 5.4 \\
Dermacryl & Octylacrylamide/acrylate copolymer & 1 & 3.5 \\
Gantrez & PVM/MA copolymer (poly(methyl vinyl ether/maleic acid)) & 1 & 2.9 \\
\hline
\end{tabular}

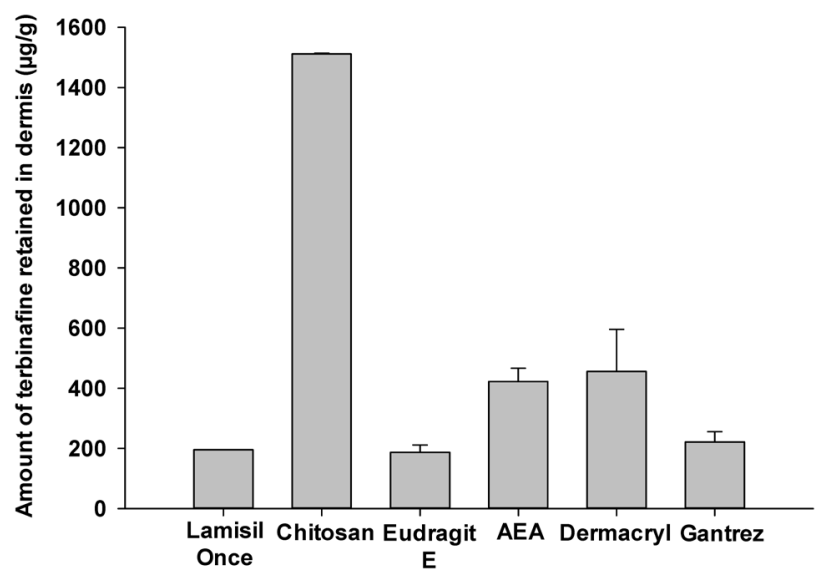

Fig. 1. Effect of Film Formers on Terbinafine Skin Retention

reported by Im et al. was used with slight modifications to produce keratinized skin in NC/Nga mice. ${ }^{14)}$ Briefly, $200 \mu \mathrm{L}$ of $1.0 \%$ 2,4-dinitrochlorobenzene (DNCB) was applied to the shaved dorsal skin of $\mathrm{NC} / \mathrm{Nga}$ mice for 4 consecutive days. Next, $200 \mu \mathrm{L}$ of $0.4 \%$ DNCB was applied to the dorsal skin that developed keratinized skin on days 8,10 , and 12. Finally, the mice were sacrificed and the keratinized dorsal skin was used for further studies. All further procedures were similar to those used for the determination of terbinafine skin permeation from different formulations.

Statistical Analysis The results are expressed as mean \pm standard deviation (S.D.) Student's $t$-test was used to determine the statistical significance of the differences between the groups. Results of $p<0.05$ were considered to be statistically significant.

\section{Results and Discussion}

Conventional topical antifungal formulations are applied once or twice daily to treat infections. However, complying with the dosing regimen and premature treatment termination are common obstacles to successful treatment, because at least 1 week, and often several weeks, may be required for effective treatment using topical antifungal formulations. In order to address these issues, topical antifungal formulations comprising terbinafine, a film-forming agent, and a solvent were prepared and evaluated.

Effect of Various Film-Forming Agents on Terbinafine Skin Retention Figure 1 shows in vitro skin retention of terbinafine with various film-forming agents, and Table 1 describes the characteristics of various film-forming solutions. Of the film-forming agents tested, chitosan showed the best capability as a film former, and it greatly enhanced terbinafine

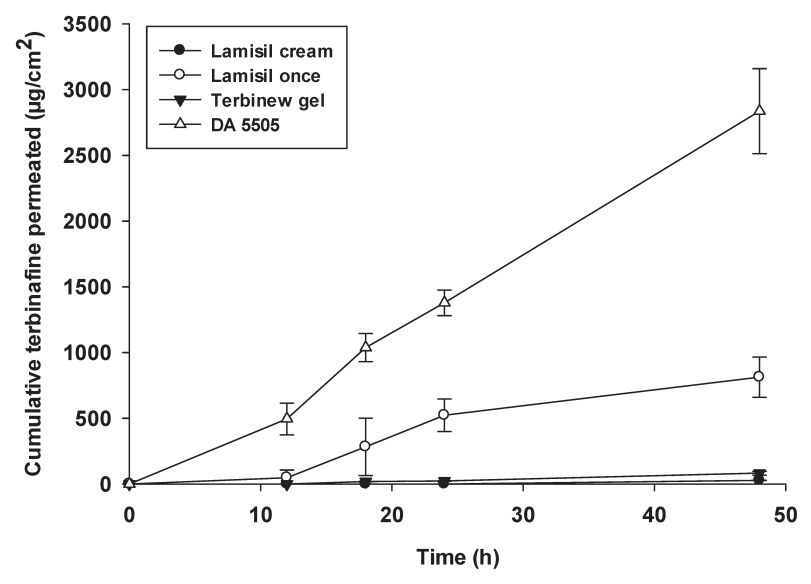

Fig. 2. Permeation of DA 5505 and Other Marketed Products into Mouse Skin

skin retention. This effect of chitosan might be a result of its film forming ability and absorption enhancing properties. ${ }^{15-17)}$ Film formation ensures that the skin is covered, which retains moisture and thus contributes to opening of the pores of the skin for drug entry. In addition, skin hydration causes swelling of keratinocytes, affecting lipid packing in the stratum corneum. Thus, initiated disruptions can cause merging of the polar and nonpolar intercellular routes, which accelerates the flux of pharmaceutical actives. ${ }^{18)}$ Furthermore, cationic chitosan interacts with the anionic skin, resulting in better skin penetration. ${ }^{11)}$ Therefore, chitosan was chosen as the best filmforming agent for the development of a topical formulation containing terbinafine.

Effect of Different Formulations of Terbinafine on Skin Permeation Figure 2 shows the in vitro skin permeation of terbinafine from different marketed formulations. Although the formulations included the same amount of terbinafine, considerable differences in permeation profiles were found. In comparison with the gel and cream formulations, the solution was found to significantly increase terbinafine skin permeation $(p<0.05)$. Among the formulations, DA 5505 produced the most terbinafine skin permeation. After $48 \mathrm{~h}$, the cumulative terbinafine permeation produced by DA 5505 was almost 100 times greater than that produced by the cream formulation, 30 times greater than that produced by the gel formulation, and 4.5 times greater than produced by the solution (Lamisil Once ${ }^{\mathbb{B}}$ ).

The skin permeation parameters of the different tested formulations are presented in Table 2. DA 5505 showed the greatest cumulative terbinafine skin permeation, which was significantly greater $(p<0.001)$ than that of the cream, gel, and solution formulations. Similarly, the flux and permeation coef- 
Table 2. Skin Permeation Parameters for Different Formulations

\begin{tabular}{lcccc}
\hline \hline Formulation & $Q_{\text {cum }}\left(\mu \mathrm{g} / \mathrm{cm}^{2}\right)$ & $J_{\text {ss }}\left(\mu \mathrm{g} / \mathrm{cm}^{2} \mathrm{~h}\right)$ & $K_{\mathrm{p}}\left(* 10^{-5} \mathrm{~cm} / \mathrm{h}\right)$ & ER of DA 5505 $(\mathrm{h})$ \\
\hline Lamisil cream & $27.7 \pm 0.8$ & $0.237 \pm 0.006$ & $0.079 \pm 0.002$ & $11.5 \pm 0.00$ \\
Terbinew gel & $82.3 \pm 14.3$ & $0.716 \pm 0.123$ & $0.238 \pm 0.041$ & $6.66 \pm 0.57$ \\
Lamisil Once ${ }^{\mathbb{R}}$ & $812.5 \pm 153.0$ & $7.217 \pm 1.585$ & $2.405 \pm 0.528$ & $3.16 \pm 3.05$ \\
DA 5505 & $2837.1 \pm 323.4^{\#, \dagger, \mathrm{t}}$ & $23.803 \pm 2.489^{\#, \dagger, \mathrm{t}}$ & $3.934 \pm 0.829^{\#, \dagger, \mathrm{t}}$ & $3.29 \pm 0.34^{c)}$ \\
\hline
\end{tabular}

Each value represents mean \pm S.D. $(n=3) .{ }^{\#} p<0.001$ compared to Lamisil cream, ${ }^{\dagger} p<0.001$ compared to Terbinew gel, ${ }^{t} p<0.001$ compared to Lamisil Once ${ }^{\circledR}$. $\left.a\right)$ Compared to Lamisil cream. b) Compared to Terbinew gel. $c$ ) Compared to Lamisil Once ${ }^{\circledR}$. Abbreviations: $Q_{\text {cum }}=$ cumulative amount of terbinafine permeated in $48 \mathrm{~h} ; J_{\text {ss }}=$ steady state flux; $K_{\mathrm{p}}=$ permeation coefficient; $\mathrm{LT}=$ lag time; $\mathrm{ER}=$ enhancement ratio.

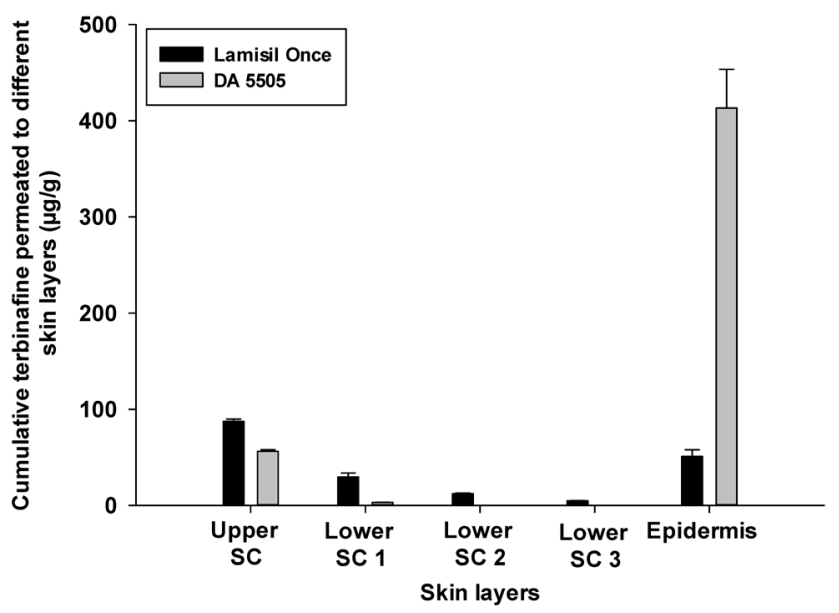

Fig. 3. Comparative Assessment of Terbinafine Permeation from Lamisil Once ${ }^{\circledR}$ and DA 5505 into Porcine Skin

ficients for DA 5505 were significantly higher than those of the other formulations $(p<0.001)$. The lag time of terbinafine permeation was significantly reduced by DA 5505 in comparison with the cream and gel formulations $(p<0.001)$. DA 5505 had an ER for terbinafine of around 100, 33, and 3 in comparison with the marketed cream, gel, and solution formulations, respectively.

The absorption of a drug into the skin tissue and the skin penetration of a drug are strongly influenced by the film-forming agent and solvent used in the drug formulation. Chitosan used in combination with DA 5505 further enhanced permeation of terbinafine through the layers of the skin. Chitosan has the potential to significantly change the secondary structure of keratin present in the stratum corneum, enhancing the water content and cell membrane fluidity to different degrees and resulting in enhanced skin permeation. ${ }^{19)}$ In addition, ethanol present in the solution might have led to enhancement of skin penetration. ${ }^{20)}$ Aqueous alcohol solutions are expected to extract proteins and lipids from the skin, resulting in minimization of the barrier function of the skin and rendering the membrane more permeable. ${ }^{21-23)}$

Comparative Assessment of Terbinafine Skin Retention in Different Skin Layers Topical fungal infections mainly invade the stratum corneum, viable epidermis, and dermis regions. Therefore, maintenance of drug concentrations in these regions is very important. The terbinafine treatment duration is very short in most cases, and sometimes a single application of the terbinafine formulation is recommended. Therefore, it is important to maintain drug concentrations in the skin layers after a single application. Figure 3 exhibits the
Table 3. Characteristics of DA 5505

\begin{tabular}{lcc}
\hline \hline \multicolumn{1}{c}{ Characteristics } & DA 5505 \\
\hline Film-forming agent & Chitosan \\
Film-forming agent's concentration $(\% \mathrm{w} / \mathrm{v})$ & & 1.0 \\
$\mathrm{pH}$ & & $4.0 \pm 0.1$ \\
Viscosity $(\mathrm{mPa}) *$ & $25 \mathrm{rpm}$ & $11857 \pm 245$ \\
& $50 \mathrm{rpm}$ & $8727 \pm 64$ \\
Clarity & $75 \mathrm{rpm}$ & $7320 \pm 183$ \\
Drug content $(\%)$ & & Clear solution \\
\hline
\end{tabular}

*Viscosity was measured using Brookfield Viscometer (No. 7 Spindle, RVDV, Brookfield, U.S.A.) at $25^{\circ} \mathrm{C}$.

comparative skin retention of terbinafine in different skin layers using DA 5505, in comparison with a commercial product (Lamisil Once ${ }^{\circledR}$ ). A chitosan-based terbinafine-loaded film forming solution formulation, DA 5505 (Table 3) produced greater epidermal and dermal skin retention of terbinafine, in comparison with the commercial product (Lamisil Once ${ }^{\circledR}$ ).

The stratum corneum is the outermost layer of the skin, and it is the skin layer that poses the greatest hindrance to skin penetration of pharmaceutical actives. Because the stratum corneum is an effective barrier to penetration of drugs into successive skin layers, ${ }^{24)}$ the drug formulation plays an important role in the penetration of drugs deep into the skin. Our results suggest that DA 5505 improved the capability of terbinafine to penetrate through the stratum corneum. Furthermore, several studies have suggested that, after terbinafine has penetrated the skin, it accumulates in the skin and persists at high concentrations for several weeks after drug application is discontinued. ${ }^{25-28)}$ Therefore, enhancement of skin penetration and retention of terbinafine by DA 5505 has the potential to enhance fungicidal effects and long-term cure rates.

Skin Retention of Terbinafine with Different Loading Doses Skin retention of antifungal agents plays a vital role in the treatment of infections. The amount of terbinafine retained in whole porcine skin after exposure to different loading doses of terbinafine is presented in Fig. 4. Lamisil Once ${ }^{\circledR}$ showed similar drug retention in whole skin at loading doses from $30 \mathrm{mg}$ to $300 \mathrm{mg}$. However, DA 5505 showed significantly increased terbinafine retention as the amount of drug loaded was increased $(p<0.05)$.

According to Fick's first law of diffusion, the concentration gradient is the major driving force for drug flux. Therefore, an increase in drug flux is expected as the loading dose of terbinafine is increased. However, this explanation is only true when there is no crystallization of the drug. As explained previ- 


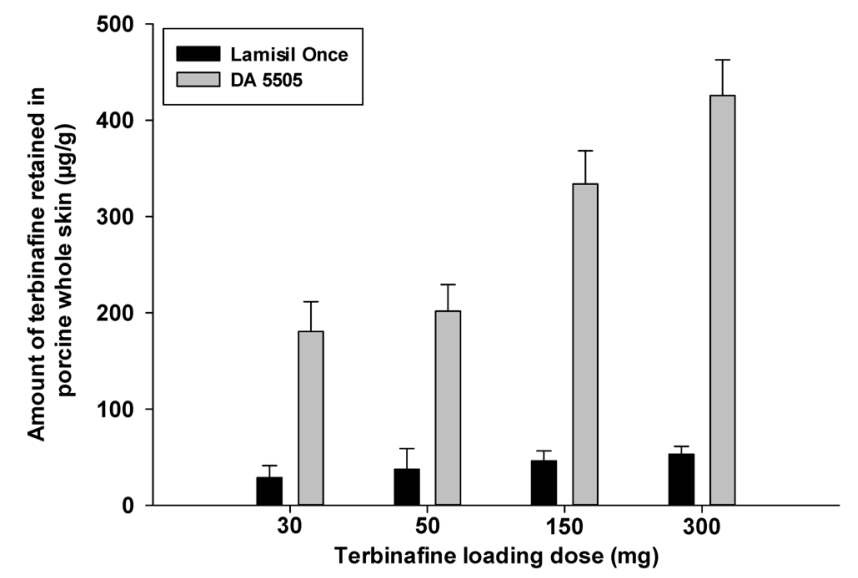

Fig. 4. Cumulative Terbinafine Retention in Whole Porcine Skin
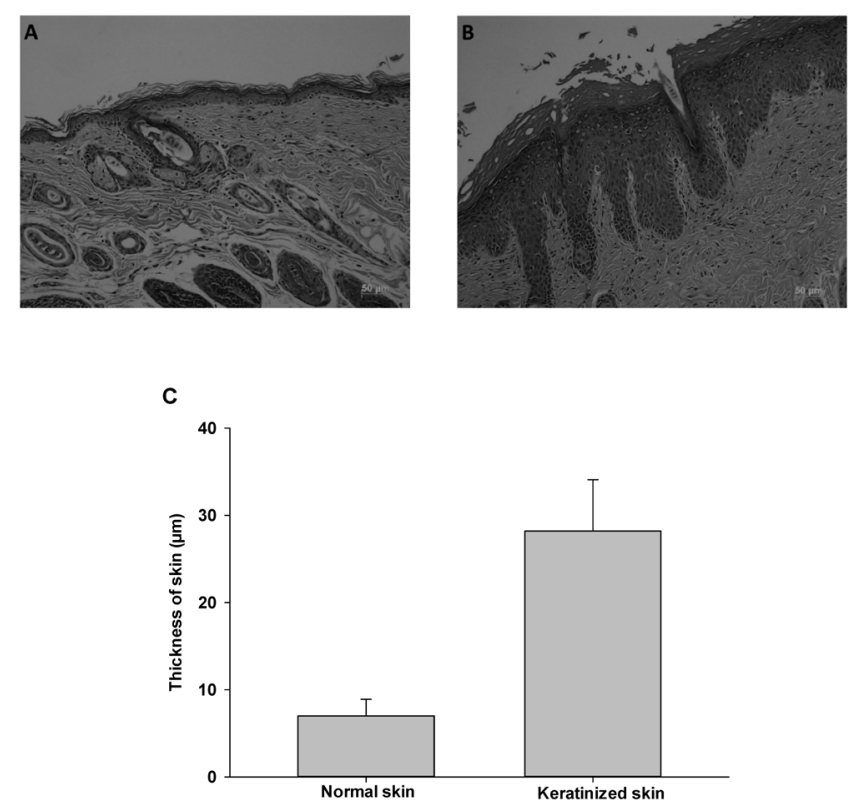

Fig. 5. Histopathological Profiles of Mouse Skin

A, normal skin; B, keratinized skin; C, thickness of normal and keratinized mouse skin.

ously, the tested formulations contained film formers. During the process of film formation, a point might be reached where the solubility of the drug is exceeded, resulting in crystallization of the drug and limiting skin permeation. Therefore, terbinafine crystallization might have limited drug permeation and retention by Lamisil Once ${ }^{\circledR}$ in comparison with DA 5505.

Formulation Effects on Terbinafine Skin Permeation The effect of formulation on terbinafine skin penetration in normal and keratinized skin was determined. For this study, keratinized skin was produced in $\mathrm{NC} / \mathrm{Nga}$ mice, which have been used as a model for atopic dermatitis because they develop atopic dermatitis-like symptoms, including excessive keratinization with thickening and drying of skin, when maintained under conventional conditions. ${ }^{29,30)}$ As presented in Fig. 5, a clear difference was observed between normal and keratinized skin. An increased number of stratum corneum layers and layer thickening were evident in keratinized skin in comparison with normal skin. Furthermore, a significant difference $(p<0.05)$ in skin thickness between normal and keratinized skin was observed. These results suggested that
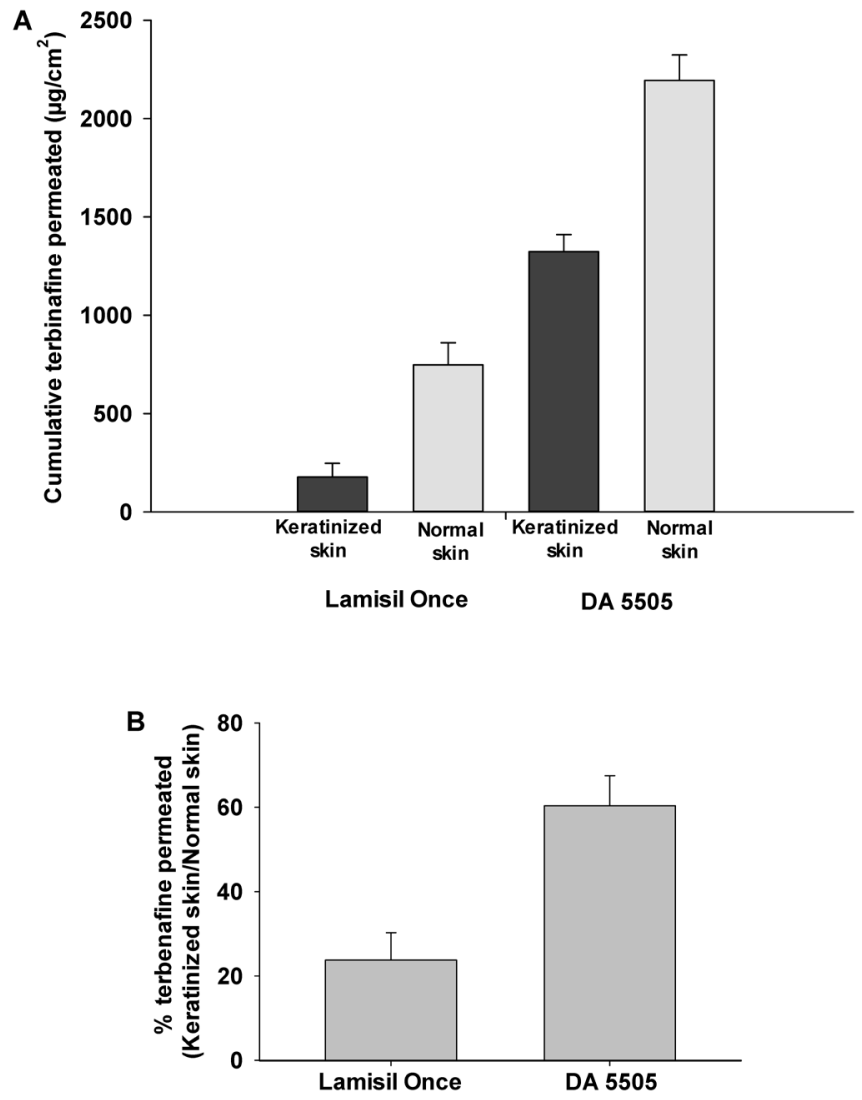

Fig. 6. (A) Terbinafine Skin Penetration in Normal and Keratinized Skin by DA 5505 and Lamisil Once ${ }^{\circledR}$; (B) Percentage of Terbinafine Permeation in Keratinized Skin in Comparison with Normal Skin

keratinized skin was successfully produced in NC/Nga mice, which were subsequently used for skin penetration and retention studies.

Figure 6A shows terbinafine skin penetration in normal and keratinized skin. Keratinized skin clearly retarded drug penetration into the skin, reducing skin permeation of the drug. A similar result was produced by the Lamisil Once ${ }^{\circledR}$ formulation. Notably, excellent terbinafine permeation was produced in normal and keratinized skin when DA 5505 was used, and permeation produced by the DA 5505 formulation was significantly greater that that produced by Lamisil Once ${ }^{\circledR}(p<0.05)$.

Alteration in stratum corneum structure during fungal infection leads to reduced skin permeability. ${ }^{31)}$ Figure $6 \mathrm{~B}$ shows the percentage of terbinafine permeation in keratinized skin in comparison with normal skin. As shown in the figure, for Lamisil Once ${ }^{\circledR}$, keratinized skin retarded the permeation to $25 \%$ of that of healthy skin, whereas DA 5505 maintained almost $60 \%$ of the skin permeation of normal skin in keratinized skin. The combined effects of ethanol and chitosan in the DA 5505 formulation might have altered keratinized cells in the skin, subsequently increasing fluidity and leading to enhanced terbinafine skin penetration.

Stability Studies The stability of the DA 5505 formulation was evaluated at periodic intervals of days to determine possible changes in appearance and drug content. DA 5505 was analyzed for a period of 3 months. No changes in the appearance of the formulation and a negligible decrease in drug content were observed after 3 months of storage at $40 \pm 2{ }^{\circ} \mathrm{C} / 75 \% \mathrm{RH}$. 


\section{Conclusion}

Topical fungal infections necessitate adequate terbinafine concentrations in the viable epidermis and dermis layers, and the stratum corneum is a barrier to drug penetration into the skin. To overcome the barrier function of the skin, which is enhanced during fungal infection, the chitosan-based formulation DA 5505 was developed. DA 5505 exhibited the potential to efficiently target terbinafine to viable skin layers. In comparison with cream and gel formulations, DA 5505, a solution formulation, showed better efficacy as a carrier for terbinafine. Therefore, terbinafine containing film-forming solutions are potential formulations for effective topical delivery of terbinafine to superficial and deep cutaneous fungal infections.

Acknowledgment This research was supported by Yeungnam University research grants in 2014.

Conflict of Interest The authors declare no conflict of interest.

\section{References}

1) Kaur I. P., Kakkar S., Expert Opin. Drug Deliv., 7, 1303-1327 (2010).

2) Çelebi N., Ermiş S., Özkan S., Drug Dev. Ind. Pharm., 41, 631-639 (2015)

3) Alberti I., Kalia Y., Naik A., Bonny J., Guy R., J. Control. Release, 71, 319-327 (2001).

4) Gupta A. K., Chow M., Daniel C. R., Aly R., Dermatol. Clin., 21, 431-462 (2003).

5) Van Duyn Graham L., Elewski B. E., Mycoses, 54, e679-e685 (2011).

6) Ryder N. S., Br. J. Dermatol., 126 (Suppl. 39), 2-7 (1992).

7) Özcan I., Abaci Ö., Uztan A. H., Aksu B., Boyacioglu H., Güneri T., Özer Ö., AAPS PharmSciTech, 10, 1024-1031 (2009).

8) Şen M., Uzun C., Güven O., Int. J. Pharm., 203, 149-157 (2000).

9) Cerchiara T., Luppi B., Bigucci F., Orienti I., Zecchi V., J. Pharm. Pharmacol., 54, 1453-1459 (2002).

10) Ravi Kumar M. N. V., React. Funct. Polym., 46, 1-27 (2000).
11) Can A., Erdal M., Gungor S., Ozsoy Y., Molecules, 18, 5455-5471 (2013).

12) Csóka I., Csányi E., Zapantis G., Nagy E., Fehér-Kiss A., Horváth G., Blazsó G., Erós I., Int. J. Pharm., 291, 11-19 (2005).

13) Erdal M. S., Peköz A. Y., Aksu B., Araman A., Pharm. Dev. Technol., 19, 565-570 (2014).

14) Im L. R., Ahn J. Y., Kim J. H., Xin M., Kwon S. U., Kim Y. K., Kim D. K., Lee Y. M., Arch. Pharm. Res., 34, 317-321 (2011).

15) Artursson P., Lindmark T., Davis S., Illum L., Pharm. Res., 11, 1358-1361 (1994).

16) Ammar H. O., Salama H. A., El-Nahhas S. A., Elmotasem H., Curr. Drug Deliv., 5, 290-298 (2008).

17) Schipper N. G. M., Olsson S., Hoogstraate J. A., deBoer A. G., Varum K. M., Artursson P., Pharm. Res., 14, 923-929 (1997).

18) Singh I., Morris A., Int. J. Pharm. Investig., 1, 4-9 (2011).

19) He W., Guo X., Xiao L., Feng M., Int. J. Pharm., 382, 234-243 (2009).

20) Lachenmeier D. W., J. Occup. Med. Toxicol., 3, 26 (2008).

21) Bommannam D., Potts R. O., Guy R. H., J. Control. Release, 16, 299-304 (1991).

22) Kai T., Mak V., Potts R., Guy R., J. Control. Release, 12, 103-112 (1990).

23) Van der Merwe D., Riviere J., Toxicol. In Vitro, 19, 69-77 (2005).

24) Güngör S., Erdal M. S., Aksu B., Journal of Cosmetics, Dermatological Sciences and Applications, 3, 56-65 (2013).

25) Faergemann J., Zehender H., Jones T., Maibach I., Acta Derm. Venereol., 71, 322-326 (1991).

26) Hill S., Thomas R., Smith S., Finlay A., Br. J. Dermatol., 127, 396-400 (1992).

27) Hosseini-Yeganeh M., Andrew J., McLachlan A., J. Pharm. Sci., 90, 1817-1828 (2001)

28) Hosseini-Yeganeh M., McLachlan A. J., Antimicrob. Agents Chemother., 46, 2219-2228 (2002).

29) Gao X. K., Nakamura N., Fuseda K., Tanaka H., Inagaki N., Nagai H., Biol. Pharm. Bull., 27, 1376-1381 (2004).

30) Suto H., Matsuda H., Mitsuishi K., Hira K., Uchida T., Unno T., Ogawa H., Ra C., Int. Arch. Allergy Immunol., 120 (Suppl. 1), 70-75 (1999).

31) Verma S., Bhardwaj A., Vij M., Bajpai P., Goutam N., Kumar L., Artif. Cells Nanomed. Biotechnol., 42, 95-101 (2014). 